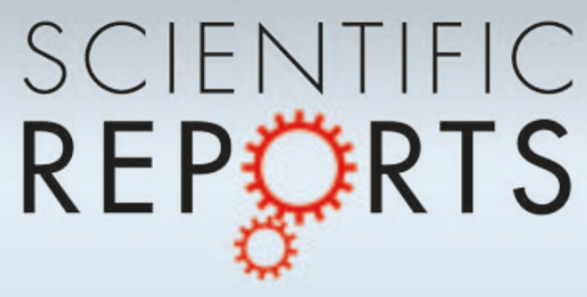

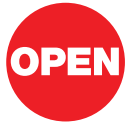

SUBJECT AREAS: SENSORS

NANOFLUIDICS NANOBIOTECHNOLOGY

ATOMIC AND MOLECULAR PHYSICS

Received

19 October 2011

Accepted

23 April 2012

Published

3 May 2012

Correspondence and requests for materials should be addressed to M.T. (taniguti@sanken. osaka-u.ac.jp)

\title{
Transverse electric field dragging of DNA in a nanochannel
}

\author{
Makusu Tsutsui, Yuhui He, Masayuki Furuhashi, Sakon Rahong, Masateru Taniguchi \& Tomoji Kawai
}

The Institute of Scientific and Industrial Research, Osaka University, 8-1 Mihogaoka, Ibaraki, Osaka 567-0047, Japan.

Nanopore analysis is an emerging single-molecule strategy for non-optical and high-throughput DNA sequencing, the principle of which is based on identification of each constituent nucleobase by measuring trans-membrane ionic current blockade or transverse tunnelling current as it moves through the pore. A crucial issue for nanopore sequencing is the fact that DNA translocates a nanopore too fast for addressing sequence with a single base resolution. Here we report that a transverse electric field can be used to slow down the translocation. We find 400 -fold decrease in the DNA translocation speed by adding a transverse field of $10 \mathrm{mV} / \mathrm{nm}$ in a gold-electrode-embedded silicon dioxide channel. The retarded flow allowed us to map the local folding pattern in individual DNA from trans-pore ionic current profiles. This field dragging approach may provide a new way to control the polynucleotide translocation kinetics.

$\mathrm{N}$ anopore sequencing is an emerging non-optical technology for high-throughput real-time single-molecule sequencing ${ }^{1-3}$. The basic idea is to identify each nucleobase by the size (or the electronic structure) through detecting a trans-pore ionic current blockade ${ }^{4-8}$ (or transverse tunnelling current ${ }^{3,9-14}$ ) during DNA translocation through a pore. A longstanding challenge for nanopore sequencing has been to slow down the flow speed of polynucleotides in the pore so as to achieve single-base spatial resolution ${ }^{6-17}$. While retarding of translocation and concomitant Ångstrom precision has recently been achieved in bioengineered nanopores by using a polymerase-DNA complex ${ }^{6}$, the system instability and limited pore-size selectivity of biological systems remains to be a critical issue for practical applications ${ }^{1}$. On the other hand, solid-state nanopores can serve as a robust and configurable single-molecule sensing platform. We present herein an electric-field dragging approach for retarding DNA translocation in a solid-state nanopore. We find that a transverse electric field of $10 \mathrm{mV} / \mathrm{nm}$ in an electrode-embedded silicon dioxide nanochannel slows down the biopolymer translocation velocity by more than two orders of magnitude. In addition, we observe field-induced unfolding of DNA. The results presented in this study suggest the usefulness of transverse field for providing essential conditions for "sequencing by tunnelling": slow translocation of unfolded DNA through an electrode gap.

\section{Results}

We measured the ionic current flowing through an electrode embedded nanochannel sensor fabricated on a $\mathrm{SiO}_{2} /$ Si wafer (Fig. 1a,b; Supplementary Fig. S1 and S2). Space between the nanoelectrodes defines the size of the fluidic channel, which is $200 \mathrm{~nm} \times 50 \mathrm{~nm} \times 60 \mathrm{~nm}$ (length $(L) \times$ width $(w) \times$ height $(h))($ Fig. 1c). These electrodes were also used to exert a transverse field on biomolecules that electrophoretically passed through the channel. A patterned polydimethylsiloxane (PDMS) block was adhered on top of the nanostructure after treating the surface with oxygen plasma (Supplementary Fig. S3). For calibration of our fluidics device, $0.1 \mathrm{M} \mathrm{KCl}$ solution buffered with $\left(\mathrm{HCl} 10 \mathrm{mM}\right.$, EDTA $1 \mathrm{mM}$ ) was injected, and ionic current across the fluidic channel $I_{\text {ion }}$ was measured under the bias voltage $V_{\text {long }}$ from $-0.5 \mathrm{~V}$ to $0.5 \mathrm{~V}$ using two $\mathrm{Ag} / \mathrm{AgCl}$ electrodes (Fig. 1d, blue plots)). The linear $I_{\text {ion }}-V_{\text {long }}$ characteristics give ionic conductance of $20.7 \mathrm{nS}$, which agrees with a theoretical estimation $e\left(\mu_{\mathrm{K}} n_{\mathrm{K}}+\mu_{\mathrm{Cl}} n_{\mathrm{Cl}}\right) h w / L=22.4 \mathrm{nS}$ (dotted line in Fig. 1d), where $\mu_{\mathrm{K}(\mathrm{Cl})}$ is the mobility of $\mathrm{K}^{+}\left(\mathrm{Cl}^{-}\right)$ions and $n_{\mathrm{K}}$ is the ion concentration. This suggests no leakage of fluid from the nanochannel. We also performed $I-V$ measurements between the embedded Au nanoelectrodes (Fig. 1d, red plots). The transverse current $I_{\text {sens }}$ was smaller than $2 \mathrm{pA}$ (below the resolution of our measurement system) over the range of transverse voltage $V_{\text {trans }}$ measured (see also Fig. S4). Two factors are presumably responsible for the low ionic conductance: the small Au surface area exposed to the solution, approximately $0.04 \mu \mathrm{m}^{2}$, and the slow charge transfer kinetics at the $\mathrm{Au}$ electrode-solution interfaces ${ }^{18}$.

Detection of a single-molecule translocation event was examined by recording $I_{\text {ion }}$ in $0.1 \mathrm{M} \mathrm{KCl}$ solution containing $10 \mathrm{nM} \lambda$-DNA (48.5 kbp) under $V_{\text {long }}=0.5 \mathrm{~V}$ at a sampling rate of $1 \mathrm{MHz}$ with no transverse field. 
a

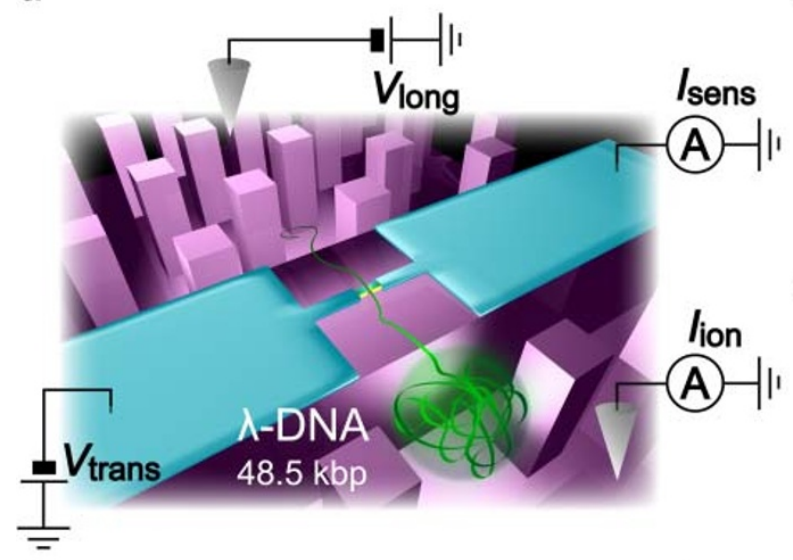

b
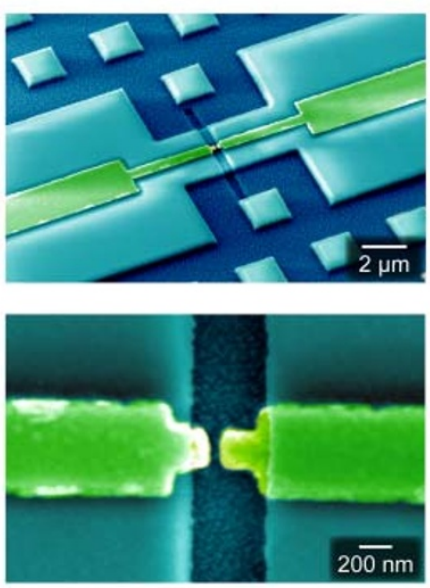

e

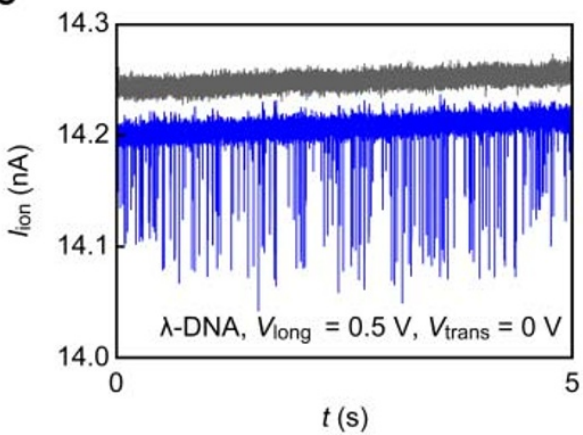

g

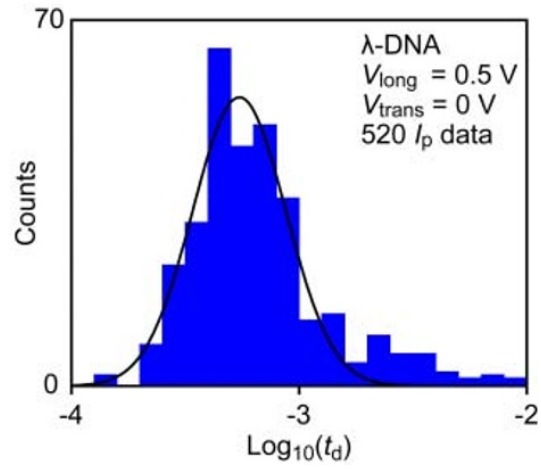

Figure 1 Electrical detection of DNA translocation in an electrode-embedded nanochannel sensor. (a) Schematic illustration of an electrodeembedded nanochannel used for single-molecule detection of DNA translocation. $48.5 \mathrm{kbp} \lambda$-DNA dissolved in a $\mathrm{KCl}$ solution is electrophoretically drawn through the electrode channel. Meanwhile, the trans-channel ionic current blockade $I_{\text {ion }}$ and the transverse current $I_{\text {sens }}$ were measured simultaneously. (b) A false-colored scanning electron micrograph of the nanochannel sensor. A pair of nanoelectrodes (yellow) is used to apply a transverse field. Micropillar arrays at both sides of the electrode channel were used as a spacer for avoiding roof collapse upon channel sealing with a PDMS block. (c) A magnified view of the nanochannel/electrode structure. The electrode gap of size $50 \mathrm{~nm} \times 60 \mathrm{~nm} \times 200 \mathrm{~nm}($ width $\times$ height $\times$ length) defines the fluidic channel dimension. (d) Ionic conductance of the electrode channel. The trans-channel ion current $I_{\text {ion }}$ measured in $0.1 \mathrm{M} \mathrm{KCl}$ solution increases nearly linearly with the driving voltage $V_{\text {long }}$ (blue plots), from which we obtained the channel conductance of $20 \mathrm{nS}$. The dotted line is the theoretical drift current flowing through the $50 \mathrm{~nm}$ channel. (e) A $I_{\text {ion }}$ trace acquired at a sampling rate of $1 \mathrm{MHz}$ in a $10 \mathrm{nM} \lambda$-DNA solution under a driving voltage of $V_{\text {long }}=0.5 \mathrm{~V}$ with no transverse field $V_{\text {trans }}$ (blue curve). Downward spike-like signals signify current blockade by polynucleotides passing through the electrode channel. Such characteristic current spikes were not observed in the control experiments conducted for the salt solution with no DNA added (gray curve). (f) A close view of a current spike, which is characterized by the magnitude of current blockade $I_{\mathrm{p}}$ and the translocation duration $t_{\mathrm{d}}$. The black and red dotted lines denote the base level current and the characteristic blockade current of $n \times 14 \mathrm{pA}(n=1,2)$ that correspond to translocation of $(n-1)$-folded DNA, respectively. The purple lines are guide to the eyes for the current steps. (g) The distribution of $t_{\mathrm{d}}$ shown in logarithmic scale. The solid line is a Gaussian peak fitted to the histogram.

Spike-like current drops were observed that were indicative of a partial ion exclusion in the nanochannel upon DNA translocation (Fig. 1e) $)^{1-4}$. This was confirmed by the control measurements in a solution containing no DNA molecules in which we found only featureless $I_{\text {ion }}-t$ curves (Fig. 1e, gray plots). The frequency for observing current spikes, or equivalently the DNA capture rate, was about $20 \mathrm{~Hz}$. Ionic current enhancement by the counteracting contribution of the counter charges of DNA molecules to the ion exclusion effect ${ }^{19}$ was not observed here. This is attributable to a substantial reduction of effective charge densities on DNA when the nanochannel size is considerably large compared to the polymer diameter ${ }^{15,19-21} . I_{\text {ion }}$ blockages are characterized by the amount of current blockage $I_{\mathrm{p}}$ and the spike width $t_{\mathrm{d}}$ (Fig. 1f). $I_{\mathrm{p}}$ 
reflects a volume of molecules that reside in the channel ${ }^{3,22}$. A rough estimation of the ionic current reduction by a volume exclusion for a stretched double-stranded DNA of $2 \mathrm{~nm}$ diameter in the channel yields $I_{\mathrm{p}}=0.014 \mathrm{nA}^{3}$. This information can be used to assess the molecular folding states as DNA with $(n-1)$ folds would produce a blockade current of $n \times 0.014 \mathrm{nA}$ (Fig. 1f) ${ }^{3,22}$. We also deduce the average translocation speed from the $t_{\mathrm{d}}$ distribution that represents DNA residence time in the nanochannel (Fig. $1 \mathrm{~g}$ ). Typical $t_{\mathrm{d}}$ is about $0.5 \mathrm{~ms}$, from which we obtain $97 \mathrm{bp} / \mu \mathrm{s}$.
Incorporation of a transverse electric field is expected to cause a substantial change in the translocation kinetics by adding a lateral electrostatic force on the negatively charged polynucleotides in the nanochannel ${ }^{23-25}$. The $I_{\text {ion }}$ curve acquired under $V_{\text {trans }}=0.5 \mathrm{~V}$ revealed DNA-associated channel blockade events at a relatively low frequency of $1.4 \mathrm{~Hz}$ (Fig. 2a). A slight increase in $I_{\text {ion }}$ base level from that at $V_{\text {trans }}=0 \mathrm{~V}$ suggests a crosstalk between the trans-channel and the transverse ionic conductance ${ }^{26}$ (see also Supplementary Fig. S5).
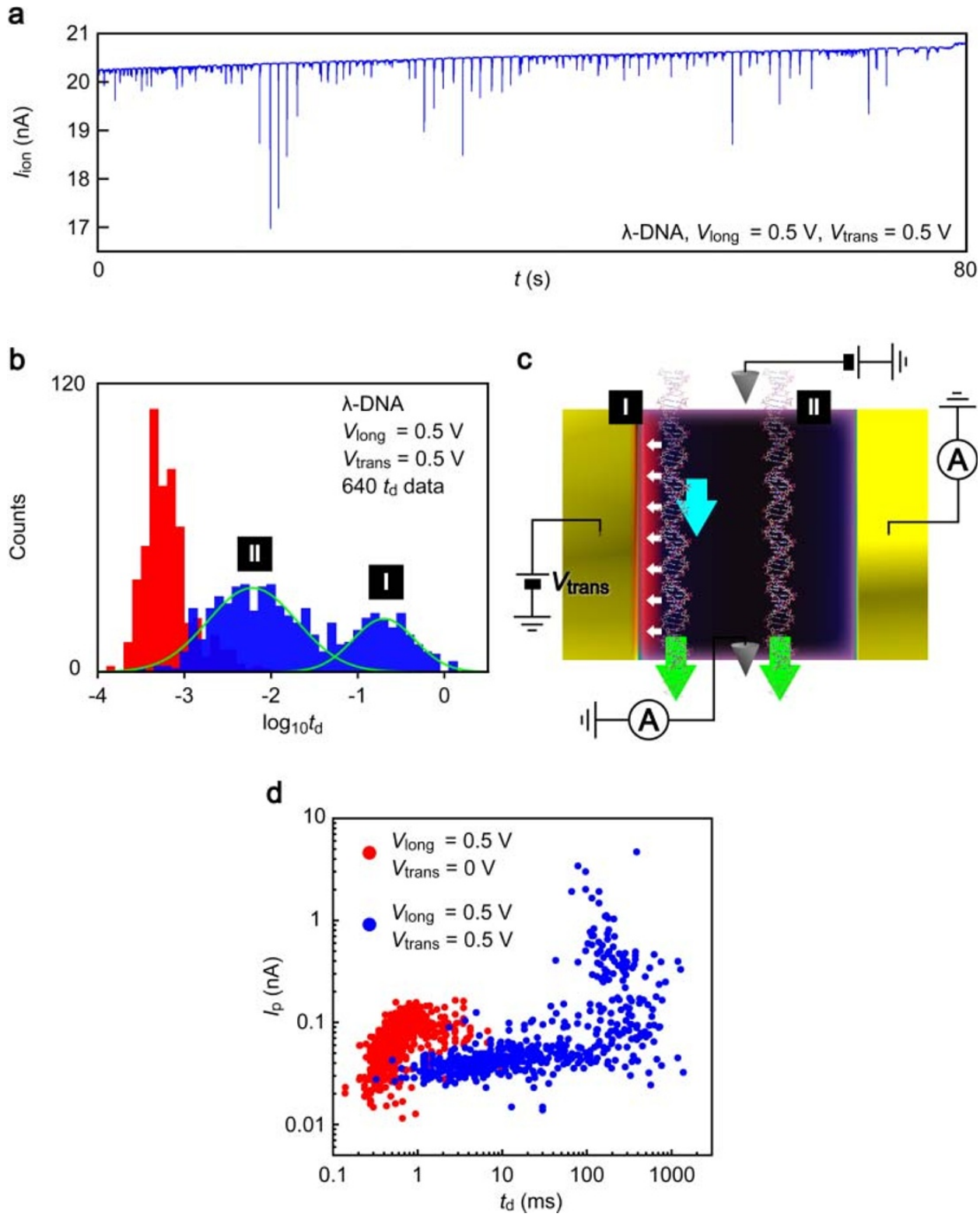

Figure $2 \mid$ Transverse electric field induced dragging of DNA in a nanochannel. (a) A $I_{\text {ion }}-t$ curve obtained in a 10 nM $\lambda$-DNA solution under $V_{\text {long }}=$ $0.5 \mathrm{~V}$ with the transverse voltage $V_{\text {trans }}=0.5 \mathrm{~V}$. Current blockades of various $I_{\mathrm{p}}$ and $t_{\mathrm{d}}$ were observed. The event frequency, or the DNA capture rate, was $1.4 \mathrm{~Hz}$. (b) The trapping duration histogram constructed with $640 t_{\mathrm{d}}$ data extracted from current blockade events detected at $V_{\text {trans }}=0.5 \mathrm{~V}$ (blue). Data for $V_{\text {trans }}=0 \mathrm{~V}$ are also displayed for comparison (red). $t_{\mathrm{d}}$ is shown in a logarithmic scale. Solid curves are the Gaussian fit to the histogram. (c) DNA translocation kinetics in an electrode-embedded nanochannel with a transverse field. Green arrows denote electrostatic forces on DNA with $V_{\text {long }}=0.5 \mathrm{~V}$ applied along the channel. (I) Polynucleotides tends to be attracted to the positively charged electrode wall when enter the positive side of the channel (white arrows). In this case, strong affinity between nucleotides and gold induces large friction against DNA permeation that more than cancels the forward electroosmotic drag force (sky blue arrow). (II) On the other hand, many polynucleotide chains translocate at around center of the nanochannel as the transverse trapping field is screened in the electrolyte solution. (d) The blockade current $I_{\mathrm{p}}$ versus the translocation duration $t_{\mathrm{d}}$ scatter plots for $V_{\text {trans }}=0 \mathrm{~V}$ (red) and $V_{\text {trans }}=0.5 \mathrm{~V}$ (blue). The constant $I_{\mathrm{p}}$ band at around $30 \mathrm{pA}$ that extends for $t_{\mathrm{d}}>100 \mathrm{~ms}$ is characteristic of molecular translocation events involving transverse-field-induced DNA-electrode interaction ${ }^{26}$. In addition, the $I_{\mathrm{p}} \propto t_{\mathrm{d}}$ relation at $V_{\text {trans }}=0 \mathrm{~V}$ cannot be explained by the constant event charge deficit model ${ }^{19}$. This indicates that DNA-electrode interaction already plays a role on the translocation at $V_{\text {trans }}=0 \mathrm{~V}^{25}$. 
To shed light on the transverse field effects on DNA translocation, a $t_{\mathrm{d}}$ histogram is constructed for the $I_{\text {ion }}-t$ curve obtained at $V_{\text {trans }}=$ $0.5 \mathrm{~V}$ (Fig. 2b). We find two peaks at $6 \mathrm{~ms}$ and $200 \mathrm{~ms}$, which correspond to the translocation speed of $8.1 \mathrm{bp} / \mu \mathrm{s}$ and $0.24 \mathrm{bp} / \mu \mathrm{s}$, respectively; the latter indicates more than two orders of magnitude reduction from the DNA velocity at $V_{\text {trans }}=0 \mathrm{~V}$. One possible explanation for the bimodal distribution is the existence of two distinct molecular pathways in the nanochannel with anti-parallel electroosmotic flow (Fig. 2c and Fig. S7). Negatively charged polynucleotides are anticipated to be attracted to the positive side of the embedded electrodes once a part of the strand approaches the electrode surface via Brownian motion, and are forced to flow within a confined nanospace defined by the screening length in $0.1 \mathrm{M} \mathrm{KCl}$ solution along the channel wall where there is forward electroosmotic dragging that facilitates the translocation ${ }^{15,24,25}$ (Fig. 2c, I). The polymer is also given an increased chance to experience large friction against the electrophoretic driving force via the electrically-induced Au electrode-DNA interactions $s^{20,27-29}$. This transverse field induced resistance overwhelms the anionic electroosmotic dragging considering the strong nonspecific affinity of DNA to $\mathrm{Au}^{11,27-30}$ as well as the electrostatic interaction between the DNA and the positively charged electrode, and causes the orders of magnitude decrease in the translocation velocity. On the other hand, the spatially confined trapping field allows large number of polynucleotide chains to electrophoretically flow at center of the nanochannel (Fig. 2c, II). These two hydrodynamic mechanisms account for the two distinct $t_{\mathrm{d}}$ states observed in Fig. 2b. Further theoretical and experimental insights are required to identify the actual DNA translocation dynamics in the nanochannel.

The role of the transverse field for reducing a DNA translocation speed is clearly shown by the scattering plot in Fig. $2 \mathrm{~d}$. It is noticeable that there is a region where $I_{\mathrm{p}}$ is almost constant while $t_{\mathrm{d}}$ varies extensively for the blockade events at $V_{\text {trans }}=0.5 \mathrm{~V}$. The low $-I_{\mathrm{p}}$ band was also observed in the recent nanopore experiments, which was explained by a cause of polynucleotide-pore interactions ${ }^{8,16}$. This further implies a contribution of field-induced Au-DNA interaction on the long translocation times. It is also inferred from the figure that the transverse field has another function to unfold DNA as large portion of the plots at $V_{\text {trans }}=0.5 \mathrm{~V}$ are clustered at around the non-folded $I_{\mathrm{p}}$ level of $20 \mathrm{pA}$.

The DNA translocation events were simultaneously detected by $I_{\text {sens }}$ measurements (Fig. 3a). $I_{\text {ion }}$ and $I_{\text {sens }}$ display a synchronous variation where the former is blocking and the latter is enhancing ${ }^{13}$ (Fig. 3b, see also Supplementary Fig. S8). Moreover, the histogram of $I_{\text {sens }}$ demonstrates two broad peaks at integer multiples of $36 \mathrm{pA}$ (Fig. 3c) that may correspond to transverse ionic conductance states in the channel occupied with zero- and one-fold DNA (Supplementary Fig. S9).

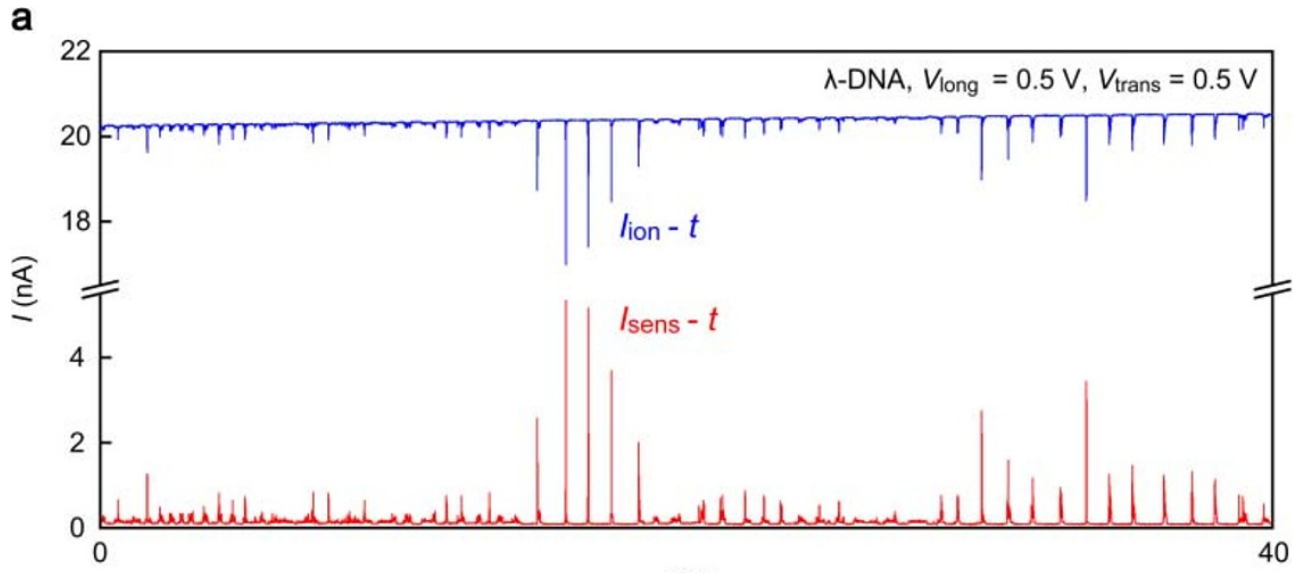

$t(\mathrm{~s})$

b

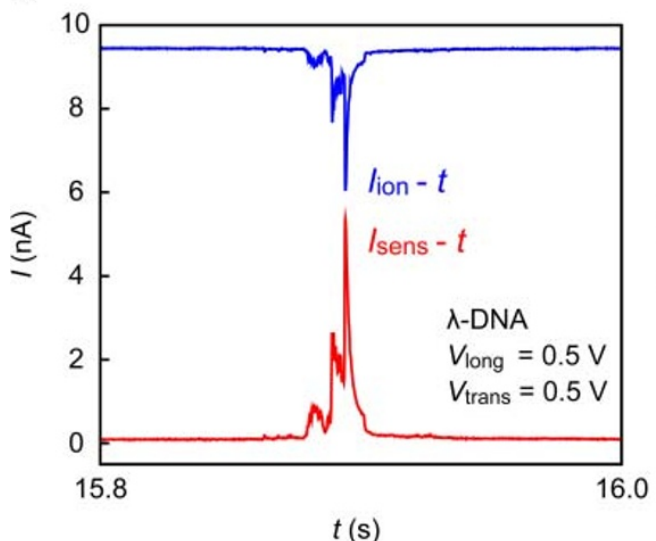

C

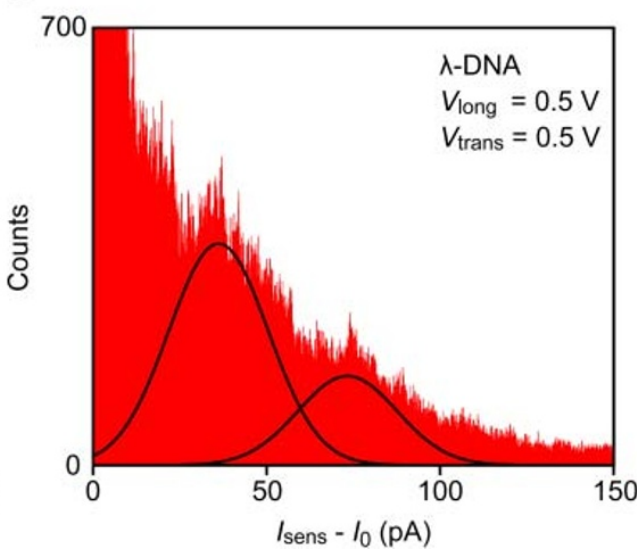

Figure $3 \mid$ Crosstalk between trans-channel and transverse ionic pathways. (a) The transverse current $I_{\text {sens }}$ concurrently measured with $I_{\text {ion }}$ under $V_{\text {long }}$ $=0.5 \mathrm{~V}$ and $V_{\text {trans }}=0.5 \mathrm{~V}$ conditions. The background current is about $100 \mathrm{pA}$. Positive pulse-like signals were found instead of current blockades. (b) Direct comparison of the $I_{\text {ion }}$ blockade and simultaneously detected $I_{\text {sens }}$ spike. The line shapes of these curves are almost identical to each other though the sign is opposite. c, A current histogram built with the $I_{\text {sens }}-t$ curve. The base level $I_{0}$ is subtracted from $I_{\text {sens }}$. Solid curves are Gaussian distributions centered at $36 \mathrm{pA}$ and $73 \mathrm{pA}$. 


\section{Discussion}

The positive $I_{\text {sens }}$ transients are obviously too large for tunnelling current flowing through the $50 \mathrm{~nm}$ gap. Instead, it is attributable to the $n$-folded ion density in the charge-induced electric double layer of DNA with $n$ folds ${ }^{31}$ that provides increased number of current-carrying ions across the transverse electrodes. Nevertheless, it is also probable that interaction between $I_{\text {ion }}$ and $I_{\text {sens }}$ demonstrated by the non-negligible influence of the trans-channel electric field on the steady-state transverse current (Supplementary Fig. S6) may also be a cause of the concomitant rise of the transverse current considering the very similar line shapes of $I_{\text {ion }}$ and $I_{\text {sens }}$ signals ${ }^{27}$. While the ability of the transverse field to slow down DNA translocation may greatly facilitates achieving sequencing by tunnelling current, the crosstalk between the trans-channel and transverse ion passages needs to be avoided, as the weak tunnelling current signatures, typically lower than $100 \mathrm{pA}$, would be buried in the transmitted ionic current blockade signals. However, the smaller the nanochannel or nanopore becomes, the lower the ionic conductance. The ion conductivity is also adjustable by the salt concentrations. Therefore, the coupling issue is expected to be resolved by employing appropriate experimental conditions to minimize the ionic current contributions to the transverse tunnelling components.

The field-retarded translocation improves the spatial resolution of nanopore detection. We observed $I_{\text {ion }}$ signals with long plateaus and steps at a multiple integer level of 20 pA (Fig. 4a). In fact, we find a pronounced peak and a shoulder in addition to the base level peak at zero in a histogram constructed with the $I_{\text {ion }}$ trace after subtracting the quasi-linear background current (Fig. 4b). The peak profile can be fitted with two Gaussian distributions at $20 \mathrm{pA}$ and $41 \mathrm{pA}$ (red curves in Fig. 4b). This peculiar feature indicates the existence of distinct DNA conformations. The magnitude of current blockage is roughly given by the channel versus molecule volume ratio ${ }^{3}$. For completely stretched double-stranded DNA of diameter $2 \mathrm{~nm}$, this

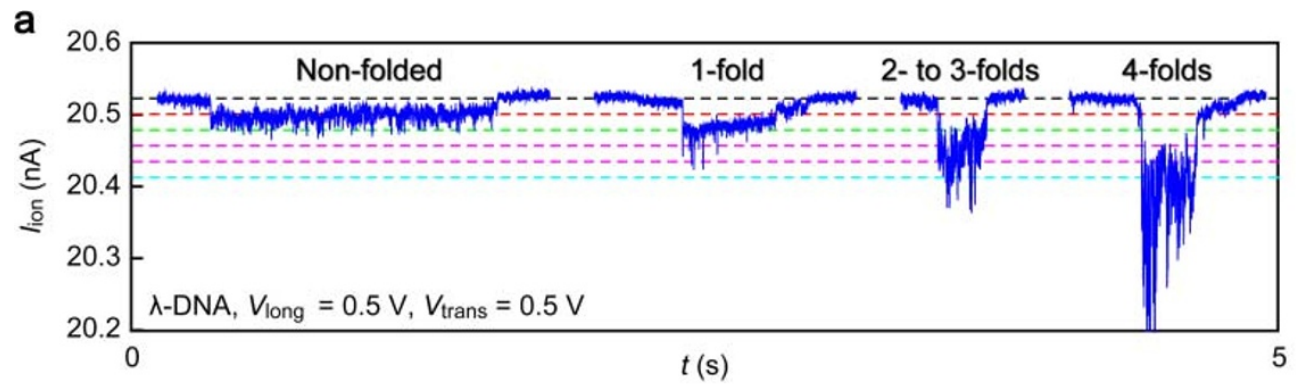

b

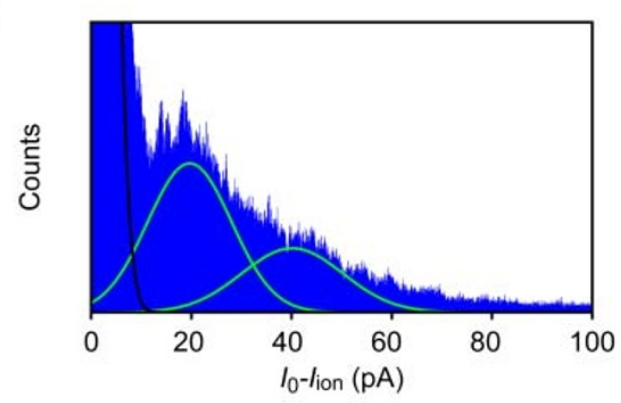

C

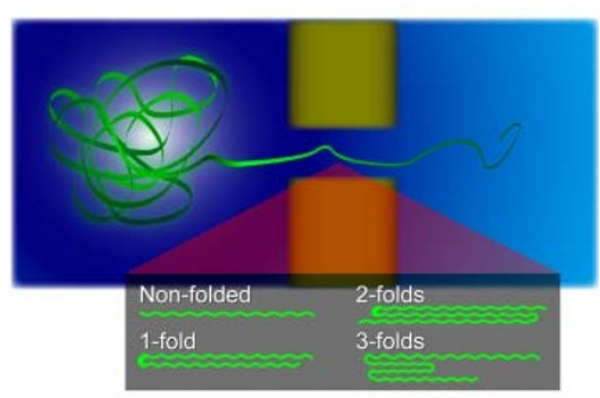

d

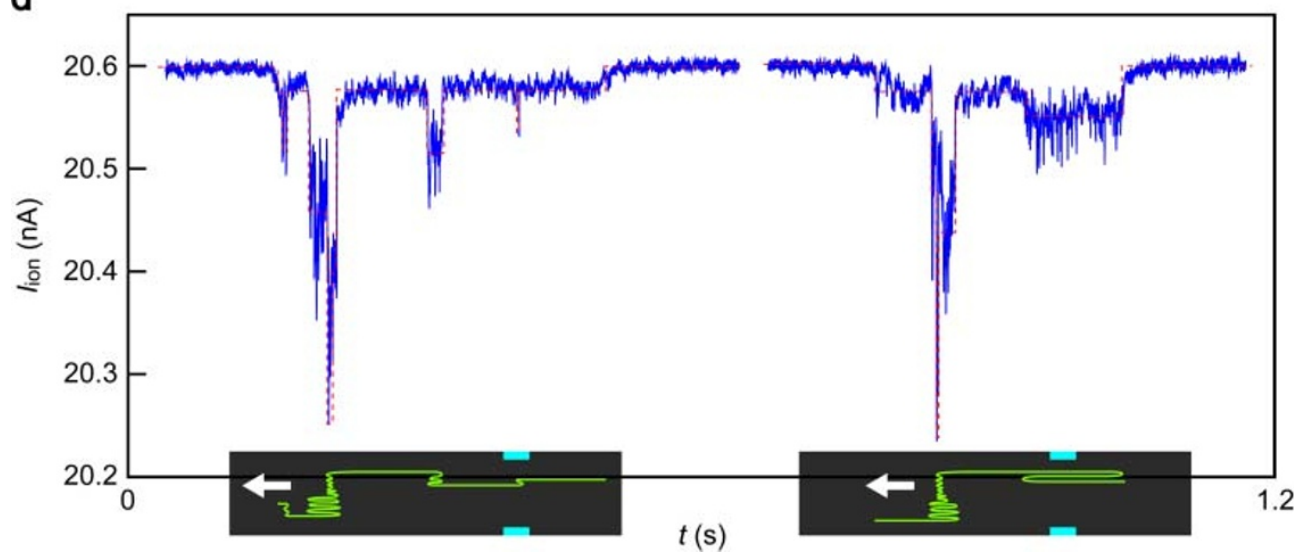

Figure $4 \mid$ Real time electrical identification of local folds in a single-molecule DNA. (a) Magnified views of long events with plateaus at $20 \mathrm{pA} \times n(n=$ $1,2,3 \cdots)$ that correspond to translocation of DNA with $n$-folds through the channel. (b) A current histogram constructed with the $I_{\text {ion }}$ trace. The background current $I_{0}$ is subtracted from $I_{\text {ion. }}$. Black line is a fit to the pronounced peak at 0 A that originates from the base level current with 10 pA noise. Green lines are the Gaussian fits to the two peaks observed at $20 \mathrm{pA}$ and $41 \mathrm{pA}$. These current states are attributed to ion blockade by non- and singlefolded DNA in the channel. (c) Schematic illustration depicting translocation of DNA having some folds. (d) Examples of identification of local folds in an individual DNA by the $I_{\text {ion }}$ landscape. The schematic models describe DNA topologies assessed from the $I_{\text {ion }}$ profiles. Red dotted lines are guide to the eyes for $I_{\text {ion }}$ steps at integer multiples of $20 \mathrm{pA}$. White arrows indicate direction of DNA flow through a nanochannel. 
ratio is $0.1 \%$. As the base level current is in a range of 20 to $21 \mathrm{nA}$, the blockade should be as large as $20 \mathrm{pA}$. When DNA is folded one time, the effective volume inside the channel doubles and so does the blockade current ${ }^{3,32}$. By analogy, the characteristic current states at $20 \times n \mathrm{pA}(n=1,2,3 \cdots)$ can thus be assigned to DNA molecules with $(n-1)$ folds (Fig. $4 \mathrm{a}, \mathrm{c})^{32}$. This enables the identification of the local folding states within an individual polynucleotide chain by referring to the plateau current levels (Fig. 4d). Fluorescence imaging of stained DNA in a nanochannel has been reported to give similar results $^{33}$. Nonetheless, the real-time single-molecule profiling capability demonstrated here is not only an electric analogue of fluorescence microscopy but with better time resolution. With further efforts to clarify the influence of the transverse field on DNA conformations and current blockages, it may find applications as a useful tool for studying atomistic biomolecule dynamics in a confined nanospace.

\section{Methods}

Fabrication of electrode-embedded nanochannels. A $0.5 \mathrm{~mm}$ thick $30 \mathrm{~mm}$ square piece of substrate was cut from a silicon wafer coated with a $700 \mathrm{~nm}$ thick thermally grown $\mathrm{SiO}_{2}$ layer. Microelectrodes were patterned on the $\mathrm{SiO}_{2}$ surface by using a photolithography method with a photo-resist AZ5206E. Subsequently, Pt $(2 \mathrm{~nm}) / \mathrm{Au}(30 \mathrm{~nm}) / \mathrm{Pt}(2 \mathrm{~nm})$ layer was deposited by a radio-frequency (RF) magnetron sputtering. The thin Pt films served as adhesion layers. The sample was then immersed in a N,N-dimethylformamide for 8 hours and sonicated for several minutes for lift-off. Following the preparation of microscale electrodes, we delineated a pair of nanoelectrodes using an electron beam (EB) lithography technique with a resist ZEP520A-7 utilizing L-shaped pattern as alignment markers. After that, $\mathrm{Pt}(2 \mathrm{~nm}) / \mathrm{Au}(30 \mathrm{~nm}) / \mathrm{Pt}(2 \mathrm{~nm}) / \mathrm{SiO}_{2}(30 \mathrm{~nm})$ multi-layered film was deposited by the RF sputtering. As a result, we obtained an electrode gap of size $200 \mathrm{~nm} \times 64 \mathrm{~nm} \times 50 \mathrm{~nm}$ (length $\times$ height $\times$ width) after the lift-off process. Furthermore, a two-leveled fluidic channel was fabricated by first depositing $\mathrm{SiO}_{2}(30 \mathrm{~nm}) / \mathrm{Cr}(100 \mathrm{~nm})$ layer on the substrate. Arrays of squares were then patterned at the both sides of the nanoelectrodes using the EB lithography processes. An etching mask was obtained by wet-etching the exposed $\mathrm{Cr}$ region in a in a diluted hydrochloric acid solution of ceric ammonium nitrate (15 wt\%). The sample was exposed to deep isotropic reactive ion etching $\left(\mathrm{CF}_{4}\right.$, $100 \mathrm{~W}$ ) to etch out $\mathrm{SiO}_{2}$. By removing the residual Cr layer, we acquired micropillars of $500 \mathrm{~nm}$ height. These posts were used as a spacer for circumventing roof collapse during PDMS sealing. Next, we again deposited $\mathrm{Cr}(25 \mathrm{~nm})$ and made a mask for a nanochannel using the RF sputtering deposition, EB lithography, and wet etching processes. Finally, the substrate was dry-etched to form a $400 \mathrm{~nm}$ channel with $60 \mathrm{~nm}$ height. The fabrication scheme is summarized in Supplementary Fig. S2.

Nanochannel sealing. SU-8 photo-resist was spin-coated on a Si wafer and baked at $95^{\circ} \mathrm{C}$ for 45 minutes. An SU-8 mold for making PDMS fluidic channels was then obtained by using a photolithography method. After that, PDMS (SYLGARD184) was cured on the mold at $70^{\circ} \mathrm{C}$ for 1 hour. As a result, a PDMS block with two grooves of $0.4 \mathrm{~mm}$ width formed at the bottom was acquired. Between the grooves had a gap of size $0.2 \mathrm{~mm}$, which was used to seal the electrode channel structure formed on a Si substrate. The sealing was implemented by treating the surface of the sample substrate and the PDMS block with oxygen plasma for activation and subsequently attached to each other to form permanent bonds.

Ionic current measurements. A tris- $\mathrm{HCl} 10 \mathrm{mM}$, EDTA $1 \mathrm{mM}$ buffer solution was prepared with Milli-Q water (Milli-pore). We used this to obtain a $0.1 \mathrm{M} \mathrm{KCl}$ solution of $\mathrm{pH}=8.0$. For single-molecule translocation detection, $48.5 \mathrm{kbp} \lambda$-DNA (Takara Bio) was added at a concentration of $10 \mathrm{nM}$. The salt solution was introduced into the channel via holes drilled in the PDMS block attached on the sample. $\mathrm{Ag} / \mathrm{AgCl}$ electrodes (BAS) inserted into additional two holes at both sides of the channel were used to measure the ionic current flowing through the electrode channel. Two cables were connected to the electrode pads for monitoring transverse currents across the nanoelectrodes. Measurements of the trans-channel and the transverse ionic current were performed by using a home-built preamplifier and a digitizer PXI-5922 (National Instruments) at a sampling rate of $1 \mathrm{MHz}$.

1. Dekker, C. Solid-state nanopores. Nat. Nanotechnol. 2, 209-215 (2007).

2. Branton, D. et al The potential and challenges of nanopore sequencing. Nat. Biotech. 26, 1146-1153 (2008).

3. Zwolak, M. \& Di Ventra, M. Colloquim: Physical approaches to DNA sequencing and detection. Rev. Mod. Phys. 80, 141-165 (2008).

4. Kasianowicz, J. J., Brandin, E., Branton, D. \& Deamer, D. W. Characterization of individual polynucleotide molecules using a membrane channel. Proc. Nat. Acad. Sci. 93, 13770-13773 (1996).
5. Clarke, J., Wu, H.-C., Jayasinghe, L., Patel, A., Reid, S. \& Bayley, H. Continuous base identification for single-molecule nanopore DNA sequencing. Nat. Nanotechnol. 4, 265-270 (2009).

6. Lieberman, K. R., Cherf, G. M., Doody, M. J., Olasagasti, F., Kolodji, Y. \& Akeson, M. Processive replication of single DNA molecules in a nanopore catalysed by phi29 DNA polymerase. J. Am. Chem. Soc. 132, 17961-17972 (2010).

7. Schneider, G. F. et al DNA translocation through graphene nanopores. Nano Lett. 10, 3163-3167 (2010).

8. Garaj, S., Hubbard, W., Reina, A., Kong, J., Branton, D. \& Golovchenko, J. A. Graphene as a subnanometre trans-electrode membrane. Nature 467, 190-193 (2010).

9. Lagerqvist, J., Zwolak, M. \& Di Ventra, M. Fast DNA sequencing via transverse electronic transport. Nano Lett. 6, 779-782 (2006).

10. Liang, X. \& Chou, S. Y. Nanogap detector inside nanofluidic channel for real-time label-free DNA analysis. Nano Lett. 8, 1472-1476 (2008).

11. Tsutsui, M., Taniguchi, M., Yokota, K. \& Kawai, T. Identifying single nucleotides by tunnelling current. Nat. Nanotechnol. 5, 286-290 (2010).

12. Huang, S. et al Identifying single bases in a DNA oligomer with electron tunnelling. Nat. Nanotechnol. 5, 868-873 (2010).

13. Ivanov, A. P. et al DNA tunneling detector embedded in a nanopore. Nano Lett. 11, 279-285 (2011)

14. Tsutsui, M., Sakon, R., Iizumi, Y., Okazaki, T., Taniguchi, M. \& Kawai, T. Singlemolecule sensing electrode embedded in-plane nanopore. Sci. Rep. 1, 46 (2011)

15. Keyser, U. F. et al Direct force measurements on DNA in a solid-state nanopore. Nat. Phys. 2, 473-477 (2006).

16. Fologea, D., Uplinger, J., Thomas, B., McNabb, D. S. \& Li, J. Slowing DNA translocation in a solid-state nanopore. Nano Lett. 5, 1734-1737 (2005).

17. Iqbal, S. M., Akin, D. \& Bashir, R. Solid-state nanopore channels with DNA selectivity. Nat. Nanotechnol. 6, 253-260 (2011).

18. Joseph, D. Bronzino, J. D. The Biomedical Engineering Handbook: Second Edition (CRC press, Boca Raton, 2000).

19. Smeets, R. M. M., Keyser, U. F., Krapf, D., Wu, M.-Y., Dekker, N. H. \& Dekker, C. Salt dependence of ion transport and DNA translocation through solid-state nanopores. Nano Lett. 6, 89-95 (2006).

20. Lu, Bo, Albertorio, F., Hoogerheide, D. P. \& Golovchenko, J. A. Origins and consequences of velocity fluctuations during DNA passage through a nanopore. Biophys. J. 101, 70-79 (2011).

21. Ravishanker, G., Auffinger, P., Langley, D. R., Jayaram, B., Young, M. A. \& Beveridge, D. L. Treatment of Counterions in Computer Simulations of DNA. Rev. Comput. Chem. 11, 317-372 (1997).

22. Fologea, D., Gershow, M., Ledden, B., McNabb, D. S., Golovchenko, J. A. \& Li, J. Detecting single stranded DNA with a solid state nanopore. Nano Lett. 5, 19051909 (2005).

23. Lagerqvist, J., Zwolak, M. \& Di Ventra, M. Influence of the environment and probes on rapid DNA sequencing via transverse electronic transport. Byophys. J. 93, 2384-2390 (2007).

24. Ai, Y., Liu, J., Zhang, B. \& Qian, S. Field effect regulation of DNA translocation through a nanopore. Anal. Chem. 82, 8217-8225 (2010).

25. He, Y., Tsutsui, M., Fan, C., Taniguchi, M. \& Kawai, T. Controlling DNA translocation through gate modulation of nanopore wall surface charges. ACS Nano 5, 5509-5518 (2011).

26. Albrecht, T. How to understand and interpret current flow in nanopore/electrode devices. ACS Nano 5, 6714-6725 (2011).

27. Takeishi, S. et al Observation of electrostatically released DNA from gold electrodes with controlled threshold voltages. J. Chem. Phys. 120, 5501 (2004).

28. Wei, R., Pedone, D., Zürner, A., Döblinger, M. \& Rant, U. Fabrication of metallized nanopores in silicon nitride membranes for single-molecule sensing. Small 6, 1406 (2010).

29. Erdmann, M., David, R., Fornof, A. R. \& Gaub, H. E. Electrically induced bonding of DNA to gold. Nat. Chem. 2, 745-749 (2010).

30. Kimura-Suda, H., Petrovykh, D. Y., Tarlov, M. J. \& Whitman, L. J. Base-dependent competitive adsorption of single-stranded DNA on gold. J. Am. Chem. Soc. 125, 9014-9015 (2003).

31. Wong, C. T. A. \& Muthukumara, M. Polymer capture by electro-osmotic flow of oppositely charged nanopores. J. Chem. Phys. 126, 164903 (2007).

32. Steinbock, L. J., Otto, O., Chimerel, C., Gornall, J. \& Keyser, U. F. Detecting DNA folding with nanocapillaries. Nano Lett. 10, 2493-2497 (2010).

33. Reccius, C. H., Stavis, S. M., Mannion, J. T., Walker, L. P. \& Craighead, H. G. Conformation, length, and speed measurements of electrodynamically stretched DNA in nanochannels. Biophys. J. 95, 273-286 (2008).

\section{Acknowledgements}

This work was supported by the Japan Society for the Promotion of Science (JSPS) through its "Funding Program for World-Leading Innovative R\&D on Science and Technology".

\section{Author contributions}

M. Tsutsui, M. Taniguchi, and T. K. planned and designed experiments. M. Tsutsui and S. R. fabricated electrode-embedded nanochannel samples. M. Tsutsui and M. Taniguchi 
exhibited ionic current measurements. M. Tsutsui, Y. H., and M. F. performed data analyses. M. Tsutsui, M. Taniguchi, and T. K. co-wrote paper.

\section{Additional information}

Supplementary information accompanies this paper at http://www.nature.com/ scientificreports
Competing financial interests: The authors declare no competing financial interests. License: This work is licensed under a Creative Commons Attribution-NonCommercial-ShareAlike 3.0 Unported License. To view a copy of this license, visit http://creativecommons.org/licenses/by-nc-sa/3.0/

How to cite this article: Tsutsui, M. et al. Transverse electric field dragging of DNA in a nanochannel. Sci. Rep. 2, 394; DOI:10.1038/srep00394 (2012). 